\title{
Performance Analysis of OBS Edge Nodes for Video Streaming
}

\author{
Felix Espina, Mikel Izal, Daniel Morató and Eduardo Magaña \\ Public University of Navarre, Campus de Arrosadía s/n, E-31006 Pamplona, Spain \\ e-mail: \{felix.espina, mikel.izal, daniel.morato, eduardo.magana\}@ unavarra.es
}

\begin{abstract}
OBS networks offer a highly efficient transport infrastructure for bursty data traffic. Video streaming content distribution networks are a clear candidate to use this promising technology. Traffic injected into an OBS network is a burst arrival process whose characteristics depend not only on input traffic parameters but also on design parameters of the OBS network. The properties of this traffic will dictate how it is affected by transmision over the OBS core and thus the user-perceived quality of the video stream that is delivered at the edge of the network. This paper evaluates different parameters in the ingress node that affect the losses suffered by the input traffic. Rules for the optimum selection of these parameters are presented: number of wavelengths, buffer size, timer value. The results show the strong dependence of losses on the timer value selected at the burst creation node.
\end{abstract}

\section{INTRODUCTION}

Optical Burst Switched (OBS) networks offer a promising optical backbone for high capacity data transport. This would benefit video streaming providers that need network infrastructures to accommodate a growing service demand. The present work addresses the dimensioning of ingress nodes for a streaming service over an OBS backbone scenario. The behavior of the output port in the ingress node is studied for an scenario of a video streaming network. This service is already being offered by Internet Service Providers (ISPs) as part of their triple or quadruple play offerings. Nowadays, the video flows are being transported over traditional ATM/SONET/MPLS networks. However, they are clear candidates to take advantage of the high transmission speeds offered by the new all-optical networks as their bandwidth consumption grows with user population and video quality (High Definition Television).

In an OBS network, the work load to the control plane is reduced by switching bursts with a large number of packets in a single operation. The packets from legacy networks are buffered at the ingress nodes and aggregated into bursts in what is often called a burstifier.

This work was funded by Spanish MEC (project STRRONG TEC2007-62192/TCM). The authors want to thank Spanish thematic network IPoTN (TEC2008-02552-E/TEC)
These bursts are optically switched by the core nodes in the network and disassembled at the egress node in order to be relayed to the destination. The prevalent burstifier types in the literature are timer-based, size-based or a mixture of both timer- and size- based [1]. In a timerbased burstifier a timer is started on the arrival of a packet to an empty burst formation queue. When the timer expires, the burst is scheduled for transmission on the output port. In a size-based burstifier the burst is sent when the planned minimum size is reached. Mixed-timer-size burstifiers complete the burst when at least one of the conditions is true: timer expiration or minimum size reached. Other less popular proposals in the literature use predictive or adaptive techniques in order to reduce the delay and losses [2], [3].

The burst formation mechanism conforms the input traffic process creating the burst traffic process that will be switched by the core nodes. Several previous works have studied the statistical properties of the burst traffic process. They typically assumed either a Poisson process or a self-similar model for the input packet traffic to the burstifier. Those studies are appropriate for general Internet traffic where a multiplex of TCP and UDP flows are present. However, the traffic process for a multiplex of real-time video streams presents different characteristics from an heterogeneous multiplex of TCP/UDP flows.

Studies such as [4], [5], [6] show that the blocking probabilities of an output port can be modeled using the Erlang-B formula due to the bufferless nature of OBS nodes. On [7] burst loss rate vs input load is analyzed by performing simulation for different burst schedulers but no buffer is assumed either. Others such as [8] address blocking probability using $\mathrm{M} / \mathrm{M} / \mathrm{k} / \mathrm{k}$ queue model in a scenario where OBS core nodes have fiber delay lines (FDL).

The performance of edge nodes in OBS networks has been studied in several works. On [9], [10], [11] a queuing model is presented for an OBS edge node. The input traffic is modeled by Markov chains and delay and throughput are studied but no results about losses are presented. In [12] the burst blocking probability of an edge node is presented but using a scenario of OBS 
with acknowledgments (OBS/A) which assumes a more circuit-like approach, thus its burstifier parameters can not be applied to a timer triggered burstifier. On [13] an edge node is studied for the case of Poisson and selfsimilar input traffic showing burst process statistics and results for buffer usage probability but the results are provided only for the 1 wavelength output port case, thus following M/G/1 models. These previous models predictions based on an Erlang-B approximation could become inadequate for future specific video distribution networks.

The performance of the transport of video flows on OBS networks has received little attention. In [14], video transmission is simulated over an OBS network, describing the effect of time- and size-triggered burstifiers but no analytical results are presented. In [15], the authors study the effect that the burst losses in the OBS network have on the decoding of video frames when the edge node uses a timer-based burstifier.

The rest of the paper is structured as follows. Section II presents the video streaming over OBS scenario under analysis and the experimental setup. Section III shows the results and discussion for a bufferless scenario showing how Erlang-B can be used to approximate losses as prelude to section IV where a more realistic finite queue scenario is explored. It is shown how edge node parameters can be chosen to get a target loss rate and how targeting user-perceived quality makes the burstifier timer a critical parameter. Finally section V concludes the paper.

\section{SCENARIO AND METODOLOGY}

The scenario under study is the network of a video on demand provider. As seen on figure 1, the provider network contains a set of video servers located in a legacy network connected through an OBS edge ingress router to the OBS core. The OBS backbone serves as a transport network for video streams that are delivered through remote edge nodes to the clients on access networks. The objective is to quantify how user-perceived quality of video depends on design parameters of the OBS architecture (such as the burstifier timeout value $T_{\text {out }}$ and the number of wavelengths used per port $c$ ). This will allow network designers to plan network resources in order to achieve a desired quality.

In a streaming process, video frames are sent in a periodic fashion following the video frame rate. There is also an intimate dependence of frames on close ones as the decoding process of a video frame normally requires the correct arrival of the packets containing the adjacent frames. The main effect of the transport of video over an OBS network is that the frame arrival process is aggregated into a burst process where the loss of a burst implies the loss of every frame transported therein.

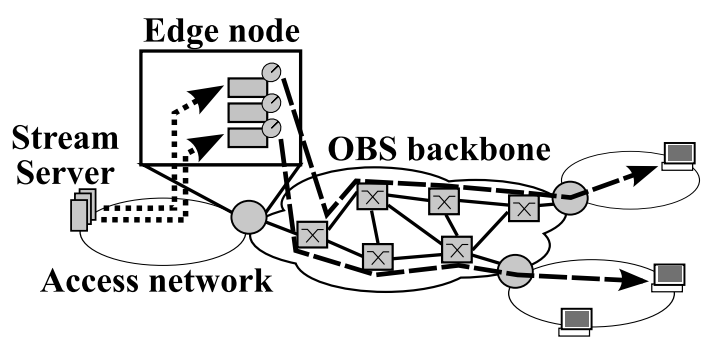

Fig. 1: Scenario

In a previous work [15] it has been studied the amount of frames that are received but cannot be decoded because they depend on frames that have already been lost. In an OBS network with timer-based burstifier every burst has the same number of frames. Consequently the fraction of lost frames is equal to the fraction of dropped busts. Thus the frame loss ratio (FLR) of a video stream whose frames are sent inside bursts is equal to burst loss probabilty (BLP). The frame starvation ratio (FSR) is defined to count as losses also undecodable frames. It has been shown how FSR approaches FLR for large values of $T_{\text {out }}$. Namely the interdependence of close video frames makes irrelevant the losses of a block of frames because most of them would be lost anyway because they cannot be decoded.

For the present work, blocking probabilities experienced at the output port of the ingress node are obtained using an ad-hoc simulator created based on the OMNeT++ framework [16]. The input traffic video traces were obtained from [17] and the results presented have been checked using movies with different bit rates, resolutions and frame rates. The aggregated high speed packet traffic used as input is the result of multiplexing a large number of video flows starting at random instants, following the behavior of requests from independent users. The video frames are transported on UDP datagrams sent from the video source. The edge node accumulates the datagrams using a different buffer for each video flow as it can be addressed to a different destination. For each video flow with a frame rate of $f_{f p s}=\frac{1}{T_{f}}$ a close to periodic burst traffic process results, with a time period related to the frame rate as $T_{b}=\left\lceil T_{\text {out }} f_{f p s}\right\rceil / f_{f p s}$ where $T_{\text {out }}$ is the timeout value in the burstifier (see Fig. 2). The number of frames in a burst is constant and given by $f_{b}=\left\lceil T_{\text {out }} f_{f p s}\right\rceil=$ $\left\lceil\frac{T_{\text {out }}}{T_{f}}\right\rceil$. The bursts created are scheduled for transmission on an empty wavelength on the output fiber link and if none is found they are queued provided there is available space in the buffer of size $b$ (or dropped in the bufferless case). 


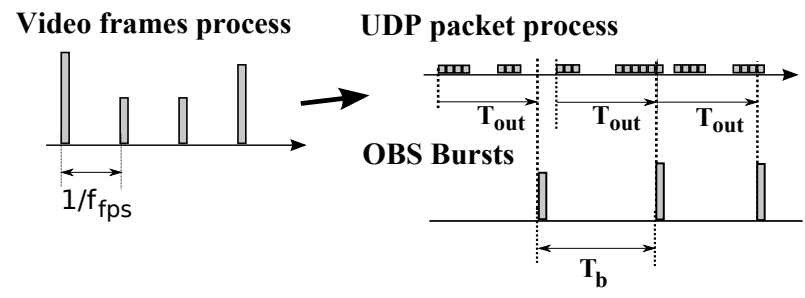

Fig. 2: Traffic flows

\section{BLOCKING PROBABILITIES IN A BUFFERLESS EDGE NODE}

In this section results for the bufferless case are examined. Fig. 3(a) presents the Burst Loss Probability (BLP) versus the output link utilization factor. The results are simulated for 32 wavelengths in the output port at $1 \mathrm{Gbps}$ each and different timer values. For comparison purposes, a reference Erlang-B formula is also presented. All the simulation runs contain more than 20 million burst arrivals and $95 \%$ confidence intervals are added to the figures. Scenarios that result in loss probabilities lower than $10^{-6}$ have not been computed because they result in unrealistic low load network configurations. Fig. 3(a) shows that the Erlang-B provides a lower bound. As the utilization factor $(\rho)$ grows, the simulations provide larger variations from the theoretical Erlang-B approximation but it is not clear from this figure the dependence on the timer value. Figure 3(b) shows the BLP versus the timer value used in the burstifier for different utilization factors. As the utilization factor grows the approximation gets worse but the quality of the estimation has not a clear dependence on the timer value.

Figure 3(c) shows the dependence of the BLP on the number of wavelengths per output port. The simulations are performed for several values of utilization factor $\rho$ and burstifier $T_{\text {out }}$ values. As can be seen, the ErlangB offers a good approximation, that improves slightly as the number of wavelengths grows. The value of $T_{\text {out }}$ has no effect on the BLP as was shown on figure 3(b), however several $T_{\text {out }}$ values are used to check, obtaining points so close that they cannot be distinguished on the figure.

The multiplexing of a large number of video flows is the reason for the convergence to an Erlang-B result. Each video flow is a periodic frame arrival process that results in a close to periodic packet arrival one (see Fig. 2). With a timer-based burstifier the burst traffic process is also close to periodic with period $T_{b}$. The video flows used to compute figure 3 are MPEG-4 flows with an average bitrate of $6.6 \mathrm{Mbps}$. In order to obtain for example an utilization factor of 0.5 with 32 wavelengths at $1 \mathrm{Gbps}$ each, 2425 video flow burst processes must be multiplexed. Each video stream generates a periodic burst arrival process, however the multiplexing of inde-
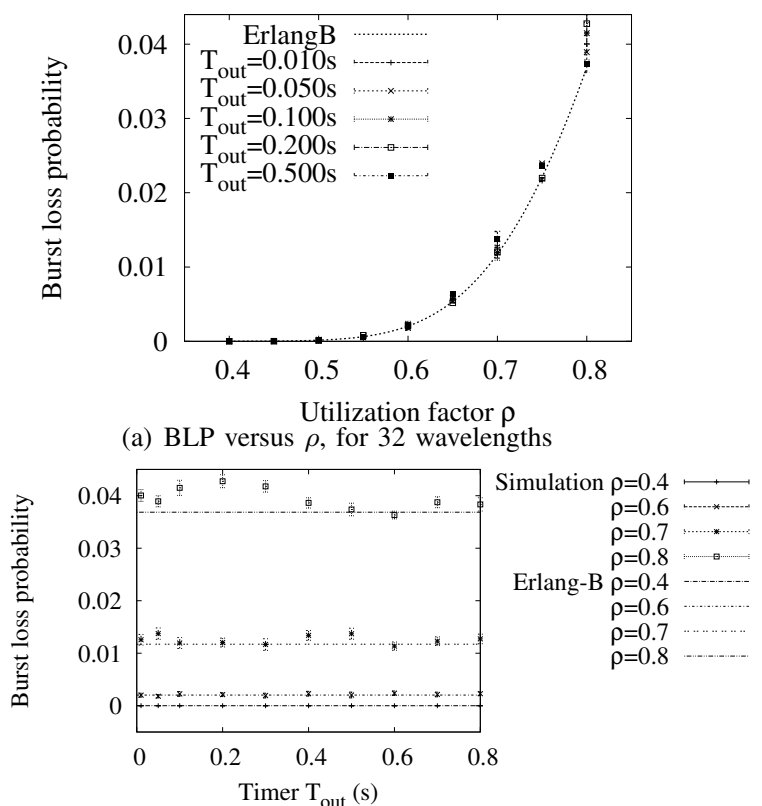

(b) BLP versus $T_{\text {out }}$, for 32 wavelengths

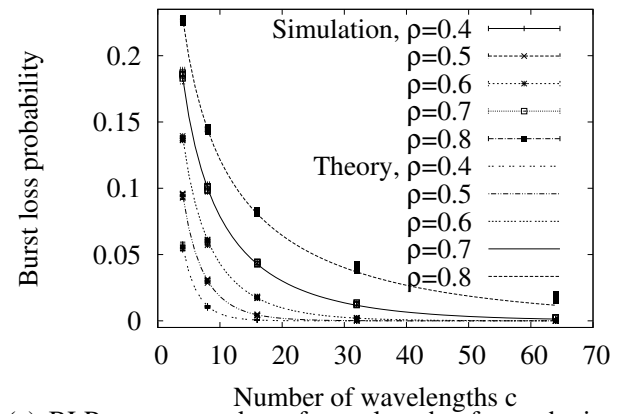

(c) BLP versus number of wavelengths, for each simulation point used $0.010 \mathrm{~s}, 0.050 \mathrm{~s}, 0.100 \mathrm{~s} 0.200 \mathrm{~s}$ and $0.500 \mathrm{~s} T_{\text {out }}$

Fig. 3: Burst loss probability (BLP) vs different parameters

pendent processes, one from each flow, results in a large number of independent arrivals in each $T_{b}$ period. For example, with 2425 video flows multiplexed there are 2425 bursts in each $T_{b}$ time, one from each flow. As the video requests from the users can be modeled as independent, the inter-arrival distribution for the multiplexed traffic is close to an exponential one during a $T_{b}$ period. Out of this interval the periodic characteristics of each burst traffic process result in periodic inter-arrival times. However, while the occupancy time of the wavelengths keeps below this time period such a periodicity has a negligible effect on the blocking probabilities and the arrival process can be considered as Poisson. The occupancy times get larger as the utilization factor grows and the result is a worse estimation as seen in Fig. 3(a).

As for the burst sizes, each video flow (also the burst process) shows Long Range Dependence, but the ErlangB formula can only be used as long as the burst size 
process can be considered a set of independent and identically distributed random variables from any distribution. A video flow presents long range dependence in the frame sizes and therefore in the burst sizes. However, as more flows are multiplexed using independent sources, samples up to the number of flows multiplexed are independent and they only show dependence with samples outside this period. Due to the large number of flows multiplexed, this period is large and has a low effect on the performance results that can assume independent burst sizes.

This discussion leads to an interest on the dependence of the results on the number of flows multiplexed. In figure 3(a) you need to multiplex 1940 flows to reach the lowest $\rho$ (0.4). Hence, only arrivals 1940-samples away show dependence and there is always an 1940large window of arrivals where the inter-arrival time is close to exponential. The number of flows could not be reduced as it would lead to negligible losses due to the low traffic intensity. However, high traffic load can be sustained even reducing the number of multiplexed flows if each flow has a large average bit rate. Figure. 4 shows how the BLP changes as the number of multiplexed flows gets reduced (going left on the x-axis). The number of wavelengths is kept at 32 , the capacity for each one at $1 \mathrm{Gbps}$ and the utilization factor at 0.7 . In order to obtain the same utilization factor for a different number of multiplexed flows the average bit rate for the flows is changed. When reducing in half the number of flows, the bit rate for each one is doubled. This is the scenario where the video quality is being increased, for example moving from a 6 Mbps MPEG-4 video flow to a 12 Mbps High-Definition flow or even higher. In order to obtain video traces with different bit rates an approximation is used by increasing the average frame size from our reference 6.6 Mbps video flow. The results in Fig. 4 are compared to the value from the Erlang-B formula. The figure shows that as the number of multiplexed flows gets reduced the Erlang-B approximation becomes an overestimation of the real value. Hence, the Erlang-B is only valid for a large number of multiplexed flows where the traffic tends to a Poisson arrival process with independent burst sizes. However, from Fig. 4 it can be seen that the approximation is valid until around 300 multiplexed flows. In order to achive a 0.7 utilization factor in a link with 32 wavelengths at $1 \mathrm{Gbps}$, the average bit rate for each video flow must be above 70 Mbps. The video streams present in High Definition media (like Blu-ray) use 36-52 Mbps flows, hence, for present scenarios the approximation would still be valid and for future scenarios the likely evolution of Video on Demand system would be to increase the number of video streams.

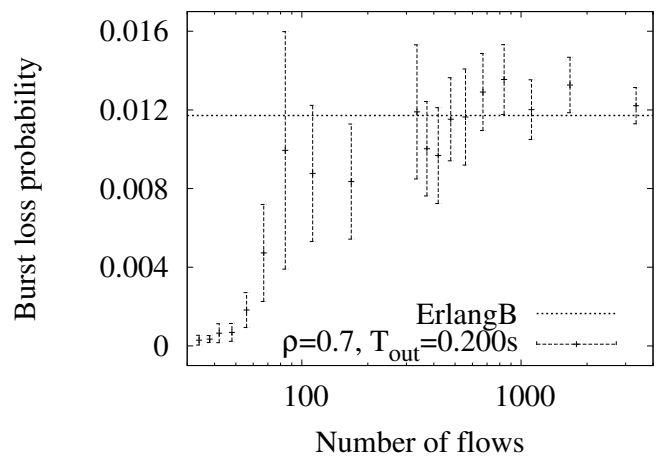

Fig. 4: Burst loss probability (BLP) versus the number of video flows, for 32 wavelengths

\section{LOSSES IN A FINITE BUFFER EDGE NODE}

In a realistic scenario the edge node architecture will include a buffer to store formed bursts while an output port wavelength becomes available. In this section we study how burst loss ratio is reduced in an edge node with buffer.

Figure 5(a) shows results of burst loss probability versus queue length for the case of $c=16, \rho=0.6$ and several values of $T_{\text {out }}$. As expected with buffer size near 0 the burst loss probability is given by Erlang-B formula $(B(c=16, c \rho=16 \times 0.6)=0.0172)$. As the buffer size grows, the loss probability decreases and it tends to 0 .

It is interesting to note that for every simulated scenario there is a buffer size value below which loss probability is near the Erlang-B value. The limit size value is different for different $T_{\text {out }}$ values. This can be explained considering that a burstifier with timer $T_{\text {out }}$ will generate bursts whose size can be modeled by a random variable $S$. It is clear that a buffer much smaller than $E[S] \approx T_{\text {out }} R$, being $R$ the stream bit rate, will not be able to store any burst, thus behaving as a bufferless system. This suggests that the buffer size may be normalized dividing it by the mean burst size $E[S]$. Plotting $\bar{b}=\frac{b}{E[S]}$ as $\mathrm{x}$ axis, every BLP curve present a similar behavior as it can be observed on figure 5(b). Regardless of $T_{\text {out }}$ value the BLP depends only on the quotient $\frac{b}{E[S]}$. It follows an exponential decay from the probability level given by the Erlang-B. This decay starts around the point where $\bar{b}=1$.

An expression for $E[S]$ can be derived from the expected frame size $E[f]$ which is given by the product of the stream bit rate and interframe time $E[f]=T_{f} R$. As the number of frames in a burst is a constant $f_{b}$, the expectation of $S$ is given by $E[S]=f_{b} E[f]=$ $\left\lceil\frac{T_{\text {out }}}{T_{f}}\right\rceil T_{f} R$

$$
\bar{b}=\frac{b}{E[S]}=\frac{b}{\left\lceil\frac{T_{o u t}}{T_{f}}\right\rceil T_{f} R}
$$




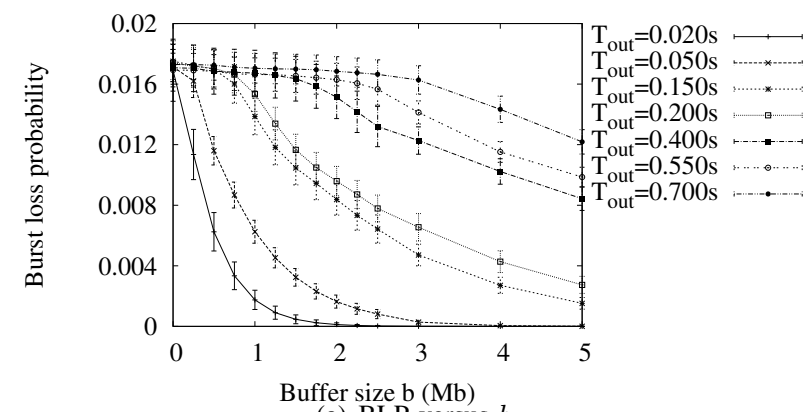

(a) BLP versus $b$

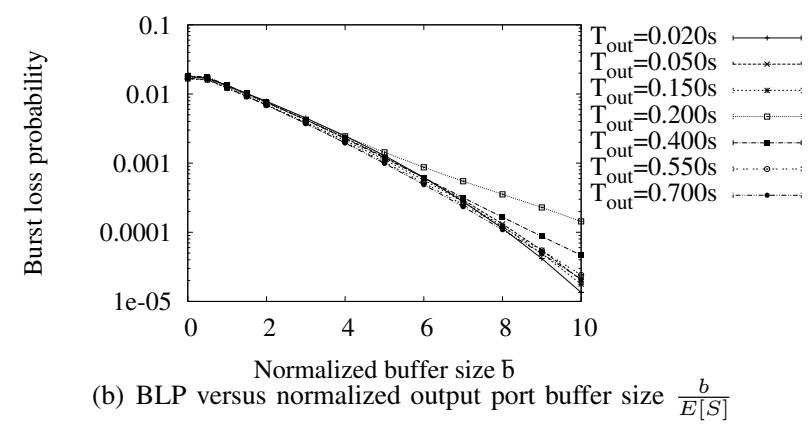

Fig. 5: Effect of output port buffer size $(b)$ on BLP, for 16 wavelengths and $0.6 \rho$

This behavior helps to dimension resources at the ingress node reducing the number of parameters needed to take into consideration. The target BLP value will impose a limit for the normalized buffer size but $T_{\text {out }}$ value remains a free parameter. On figure 6 , the value of BLP is shown versus normalized buffer size for different values of utilization factor $\rho$ and number of wavelenghts $c$. Values of $\rho$ and $c$ determine the worst case losses, given by the Erlang-B $(B(c, c \rho))$. And normalized buffer size $\bar{b}$ determines how much losses can be reduced by setting a buffer that can accommodate several bursts. BLP is approximately exponential with $\bar{b}$ but its decay rate depends strongly on $\rho$.

Let's assume an ingress node with $c$ wavelengths at the access link and with buffer size $b$. In order to plan an ingress node configuration $T_{\text {out }}$ to get a target burst loss probability BLP a curve is chosen among the plots at figure 6 that corresponds to the number of wavelengths $c$. The target BLP imposes a minimum value of buffer $\bar{b}$ to limit burst losses. As the amount of memory is limited to $b$ the restriction to $\bar{b}$ implies there is a maximum value of timer $T_{\text {out }}^{*}$ value not to surpass target loss ratio.

Thus, above parameters are enough to dimension an edge node (choosing $T_{\text {out }}, b, c$ ) for an incoming traffic $\rho$ and a target burst or frame loss probability. But as it was shown in [15] a given frame loss ratio FLR has different effect on the user-perceived quality of the received video stream depending on $T_{\text {out }}$. This is due to two causes. First the timer value determines the number of frames that are carried by a burst and therefore the number of

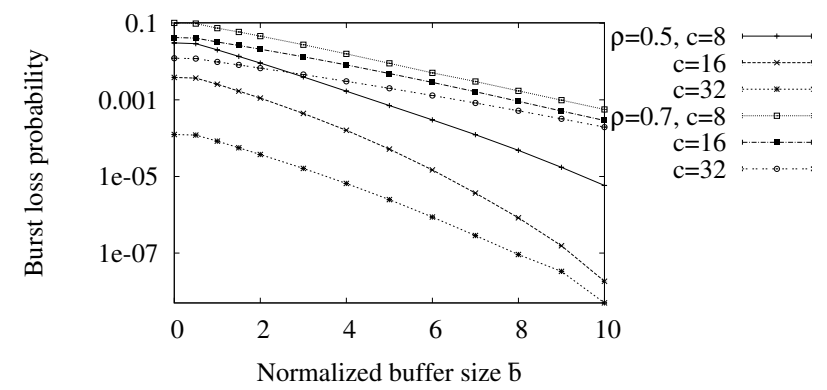

Fig. 6: Burst loss probability versus normalized output port buffer size, for $0.200 \mathrm{~s} T_{\text {out }}$

losses caused by a burst drop. Also, some frames on a video stream are coded using inter-frame codification, which means some frames contain information on the image differences to certain close frames so the loss of a frame sometimes make other frames nondecodable. To account for these extra frames losses the frame starvation ratio (FSR) is defined as the ratio of frames that can not be rendered. The user-perceived video quality is better represented by FSR.

Figure 7 shows FLR and FSR for previous case of dimensioning the $T_{\text {out }}$ value. In figure 7(a) we see how FLR depends on $T_{\text {out }}$ if we keep a constant value of buffer size. FLR decreases as $T_{\text {out }}$ decreases so we can say the values of timer lower than $T_{\text {out }}^{*}$ obtained before will get loss ratio smaller than target. But if we target a desired user-perceived quality given by a value of FSR behavior is a bit more complex. Figure 7(b) shows how FSR depends on $T_{\text {out }}$ for several values of $b$. The shape of FSR vs $T_{\text {out }}$ is not an increasing function, so finding a value of $T_{\text {out }}^{*}$ with figure 6 that achieves target FSR does not mean it is a maximum timer value.

This behavior is due to the fact that FSR combines two factors with different dependence on $T_{\text {out }}$. On one hand, as timer increases the amount of frames per burst increases.Thus, FSR gets closer to FLR because usually the frames which would be dependent on close ones are lost together on the same burst. On the other hand the actual amount of frames lost (FLR) increases with timer due to the increase of the average burst size which makes $\bar{b}$ smaller.

Figure 7(b) does just account for FSR caused by burst losses at the ingress node. However, users will receive the stream after it has traversed the OBS core where it will be subject to losses. Let's call $p_{\text {edge }}$ the burst loss probability at the edge node caused by overflow from buffer. $p_{\text {edge }}$ depends on timer as seen on figure 7(a). Trying to minimize losses at the edge suggests that the value of $T_{\text {out }}$ should be decreased below a maximum one. Let's call $p_{\text {core }}$ to burst loss probability at the OBS backbone. As OBS bursts are scheduled by switches with no buffer, the loss probability at the core depends only 


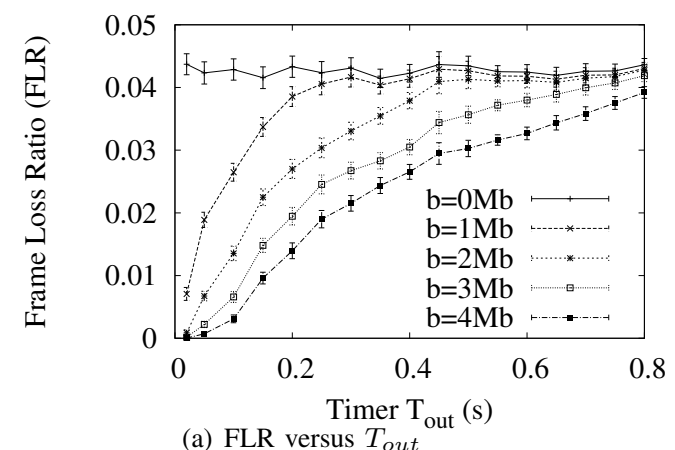

(a) FLR versus $T_{\text {out }}$

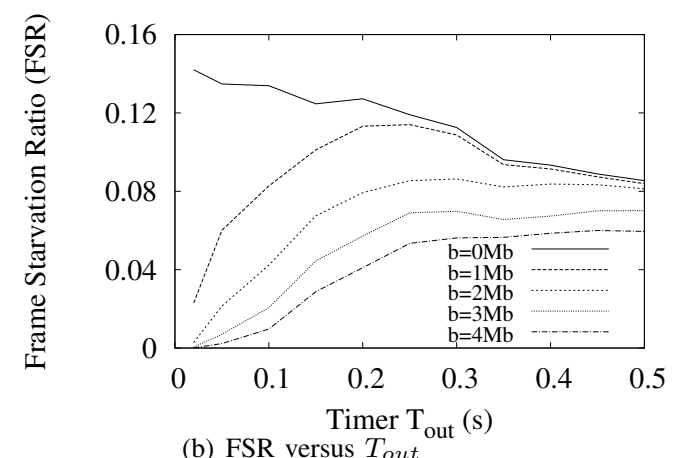

Fig. 7: Effect of taking into account the non-decoded frames, for 16 wavelengths and $0.7 \rho$

on utilization factor. This means, average burst size and $T_{\text {out }}$ have no impact on $p_{\text {core }}$. As it has been shown in [15] this suggests increasing $T_{\text {out }}$ in order to decrease FSR caused by the backbone.

Thus, the choosing of optimum $T_{\text {out }}$ value implies opposite actions to reduce losses. Larger timers are preferred at the bufferless core but smaller $T_{\text {out }}$ values should be used to increase buffer efficiency at ingress nodes. The timer value used at the burstifier becomes a critical parameter when a given user-perceived quality is desired.

\section{ConClusions}

This work has analyzed the effect of the OBS ingress node for the transmission of video streams in a video on demand scenario. It has been shown that Erlang-B formula can be used to approximate the losses introduced by a bufferles ingress node in a video over OBS scenario. In the finite buffer scenario the loss rate follows an exponential decay starting at Erlang-B values for zero buffer. Concerning resource planing of OBS ingress nodes, it has been shown that losses depend on $\rho, c$ and $\bar{b}$ (normalized buffer size obtained dividing buffer size by average burst size) and this dependency can be used to obtain a maximum burstifier timer value to obtain a target loss probability. Finally it has been shown that to dimension resources based on user-perceived quality, the impact of burstifier timer $\left(T_{\text {out }}\right)$ is strong and has to be considered as a separate parameter

\section{REFERENCES}

[1] J. Choi, J. Choi, and M. Kang, "Dimensioning burst assembly process in optical burst switching networks," IEICE Transactions on Communications, vol. E88-B(10), pp. 3855-3863, (2005).

[2] D. Morató, J. Aracil, L. Díez, M. Izal, and E. Magaña, “On linear prediction of internet traffic for packet and burst switching networks," in Proceedings of the IEEE ICCCN'01, 2001.

[3] M. Izal, J. Aracil, D. Morató, and E. Magaña, "Delay-throughput curves for timer-based obs burstifiers with light load," IEEE/OSA Journal of Lightwave Technology, 2005.

[4] K. Dolzer and C. Gauger, "On burst assembly in optical burst switched networks - a performance evaluation of justenough-time," in Proceedings of the 17th International Teletraffic Congress, 2001.

[5] X. Yu, J. Li, X. Cao, Y. Chen, and C. Qiao, "Traffic statistics and performance evaluation in optical burst switched networks," IEEE Journal of Lightwave Technology, vol. 22, no. 12, pp. 2722-2738, (2004).

[6] S. Verma, H. Chaskar, and R. Ravikanth, "Optical burst switching: a viable solution for terabit ip backbone," IEEE Network, pp. 48-53, November 2000.

[7] J. Li, C. Qiao, J. Xu, and D. Xu, "Maximizing throughput for optical burst switching networks," IEEE/ACM Transactions on Networking, vol. 15, pp. 1163-1175, October 2007.

[8] D. Morato and J. Aracil, "On the use of balking for estimation of the blocking probabilities for OBS routers with FDL lines," Lecture Notes in Computer Science, vol. 3961, pp. 308-408, 2006.

[9] L. Xu, H. G. Perros, and G. N. Rouskas, "Performance analysis of an edge optical burst switching node with a large number of wavelengths," in In 18th International Teletraffic Congress (ITC), 2003.

[10] L. Xu, H. G. Perros, and G. N. Rouskas, "A queueing network model of an edge optical burst switching node," in IEEE INFOCOM 2003, April 2003.

[11] L. Xu, H. G. Perros, and G. N. Rouskas, "Performance modeling of an edge optical burst switching node," in the 17th International Symposium on Computer and Information Sciences, (Orlando, Florida), pp. 377-381, October 2002.

[12] A. Zalesky, E. Wong, M. Zukerman, H. L. Vu, and R. Tucker, "Performance analysis of an OBS edge router," Photonics Technology Letters, IEEE, vol. 16, pp. 695-697, Feb 2004.

[13] H. Li and I. L.-J. Thng, "Edge node buffer usage in optical burst switching networks," Photonic Networks Communications, no. 13 , pp. 31-51, 2007.

[14] T. Vargas, J. Guerri, and S. Sales, "Effect and optimization of burst assembly algorithms for video traffic transmissions over OBS networks," in Proceedings of BROADNETSO8, (London, 2008).

[15] F. Espina, D. Morato, M. Izal, and E. Magaña, "The effect of burst formation timers on video streaming over optical burst switched networks," in Proceedings of BROADNETSO8, (London, 2008).

[16] A. Varga, "The OMNeT++ discrete event simulation system," in Proceedings of the European Simulation Multiconference, June 2001.

[17] G. V. der Auwera, P. T. David, and M. Reisslein, "Traffic Characteristis of H.264/AVC Variable Bit Rate Video," tech. rep., (Arizona State University, March 2007). 